\title{
A Converse to a Theorem by Friedrichs
}

\section{JoSEPH SLAwnY}

Department of Nuclear Physics, Weizmann Institute of Science, Rehovot, Israel

Received March 26, 1969

Abstract. It is proved that the requirement of implementability of a group of canonical transformations defines a class of irreducible representations of the CAR. As a corollary a converse to Friedrichs' theorem about canonical transformations implementable in the Fock representation is obtained.

A well known theorem due to K. O. FrIEDrichs [1] states that a (linear) canonical transformation

$$
b^{+}(f)=a^{+}(A f)+a^{-}(B f)
$$

is unitarily implementable in the Fock representation of the canonical anticommutation relations if, and only if, $B$ is of the Hilbert-Schmidt type (i.e.: $B^{*} B$ is of the trace class).

In this note we prove the following converse theorem: if in an irreducible representation of the canonical anticommutation relations all canonical transformations (*) with $B=0$ are implementable then it is the Fock or the anti-Fock representation.

Before going further, let us recall the definitions.

Let $H$ be separable Hilbert space (the space of the test functions). By a representation of the canonical anticommutation relations (CAR) over $H$ in a Hilbert space $\mathscr{H}$ we mean a linear mapping $a^{+}: H \rightarrow \mathscr{L}(\mathscr{H})$ such that if $a^{-}(f):=a^{+}(f)^{*}, f \in H$, then:

$a^{-}(f) a^{+}(g)+a^{+}(g) a^{-}(f)=(f \mid g) \quad$ and $a^{+}(f) a^{+}(g)+a^{+}(g) a^{+}(f)=0$.

A Fock (resp.: an anti-Fock) representation of the CAR is an irreducible representation for which there exists $\Omega \in \mathscr{H}$ such, that $a^{-}(f) \Omega=0$ (resp. : $a^{+}(f) \Omega=0$ ) for all $f \in H$.

One says that a pair $(A, B), A$ linear and $B$ an antilinear operators in $H$, defines a canonical transformation if $b^{+}$defined by $\left(^{*}\right)$ is a representation of the CAR.

If, in addition, there exists such unitary $U \in \mathscr{L}(\mathscr{H})$ that

$$
b^{+}(f)=U a^{+}(f) U^{-1}, \quad f \in H .
$$

then it is said that canonical transformation is (unitarily) implementable in the given representation. 
In Sections 1-4, we give an analysis of the following situation:

Let $S_{1}$ denote the multiplicative group of the complex numbers of modulus one, $G=S_{1} \times S_{1} \times \ldots$ and let $\left\{f_{i}\right\}_{i=1}^{\infty}$ be some orthonormal basis of $H$.

Let $g=\left(z_{1}, z_{2}, \ldots\right) \in G$ and let $A_{g}$ be such an unitary operator that $A_{g} f_{i}=z_{i} f_{i}, i=1,2, \ldots$, and $B_{g}=0$; the pair $\left(A_{g} B_{g}\right)$ defines a canonical transformation.

We will investigate the restrictions imposed on an irreducible representation of the CAR by the following condition: all the canonical transformations defined by $\left(A_{g}, B_{g}\right), g \in G$, are implementable.

By factorization, the analysis is applicable to the factor type I representations and, with taking needed care, to the representations of the canonical commutation relations too.

1. Let us choose for each $g \in G$ a unitary operator $U_{g}$ such that:

$$
a^{+}\left(A_{g} f\right)=U_{g} a^{+}(f) U_{g}^{-1}, \quad f \in H .
$$

$U_{g}$ is defined by this condition up to a factor of modulus one.

In what follows we will consider $G$ as a topological, compact and separable group: a denumerable product of the compact and separable, topological groups $S_{1}$.

In $G$ there exists a natural Borel structure generated by that topology and in this section we want to prove that for every $x, y \in \mathscr{H}$ the function $g \rightarrow\left|\left(x \mid U_{g} y\right)\right|$ is a Borel function.

Let $a_{i}^{ \pm}:=a_{i}^{ \pm}\left(f_{i}\right)$ and let $a_{i}$ denote $a_{i}^{+}$or $a_{i}^{-}$. Because of the estimation $\|a(f)\| \leqq\|f\|, f \in H,[2]$, separability of $H$ and irreducibility of our representation the Hilbert space $\mathscr{H}$ is separable and the *-algebra $\mathscr{A}$ generated by $\left\{a_{i}\right\}$ acts irreducibly in $\mathscr{H}$.

Because of $U_{g} a_{i}^{+} U_{g}^{-1}=z_{i} a_{i}^{+}, g \rightarrow U_{g} a_{i} U_{g}^{-1}$ is a norm continuous function for every $i$ and therefore for each $A \in \mathscr{A} g \rightarrow U_{g} A U_{g}^{-1}$ is norm continuous too.

Let us consider some $A \in \mathscr{L}(\mathscr{H})$. Because of the irreducibility of $\mathscr{A}$ and separability of $\mathscr{H}$ there exists a sequence $A_{n} \in \mathscr{A}$ such that $A_{n} \rightarrow A$ weakly $([3], \S 3)$.

Therefore, for $y \in \mathscr{H}, g \in G$ :

$$
\begin{aligned}
\left(y \mid U_{g} A U_{g}^{-1} y\right)=\left(U_{q}^{-1} y \mid A U_{g}^{-1} y\right) & =\lim _{n \rightarrow \infty}\left(U_{g}^{-1} y \mid A_{n} U_{g}^{-1} y\right) \\
& =\lim _{n \rightarrow \infty}\left(y \mid U_{g} A_{n} U_{g}^{-1} y\right) .
\end{aligned}
$$

Thus, the function $g \rightarrow\left(y \mid U_{g} A U_{g}^{-1} y\right)$ being a point limit of a sequence $g \rightarrow\left(y \mid U_{g} A_{n} U_{g}^{-1} y\right)$ of continuous functions, is a Borel function on $G$. Taking for $A$ the orthogonal projection on subspace generated by $x$ we have:

$$
\left(y \mid U_{g} A U_{g}^{-1} y\right)=\left|\left(y \mid U_{g} x\right)\right|^{2}
$$

and therefore for every $x, y \in \mathscr{H}, g \rightarrow\left|\left(y \mid U_{g} x\right)\right|$ is a Borel function on $G$. 
2. In this section using the very strong results of G. W. Mackey [4], (see however, the remark in the end of this section) we will prove the following.

Lemma. There exists a finite dimensional $x$ subspace of $\mathscr{H}$ invariant for all $U_{g}$.

For the proof let us proceed as follows: from Section 2, we know that $g \rightarrow\left|\left(x \mid U_{g} y\right)\right|$ is a Borel function on $G$ for all $x, y \in \mathscr{H}$. Using the method of proof of the Theorem 2.2 in [4] we may define such function $f$ on $G,|f(g)|=1$, that for every $x, y \in \mathscr{H} g \rightarrow\left(x \mid f(g) U_{g} y\right)$ is a Borel function. Now, if $V_{g}:=f(g) U_{g}$ then $g \rightarrow V_{g}$ is a projective representation of $G$ in the sence of [4] with multiplier $\sigma$ defined by: $V_{g_{1} g_{2}}=\sigma\left(g_{1}, g_{2}\right) V_{g_{1}} V_{g_{2}}$. After introducing in $S_{1} \times G$ a multiplication; $\left(\lambda_{1}, g_{1}\right)\left(\lambda_{2} g_{2}\right)=\left(\frac{\lambda_{1} \lambda_{2}}{\sigma\left(g_{1}, g_{2}\right)}, g_{1} g_{2}\right)$ one obtains a group $G^{\sigma}$ and $(\lambda, g) \rightarrow \lambda V_{g}$ is an ordinary representation of this group.

Now Theorem 2.1 of [4] asserts that in $G$ there exists a topology that makes $G^{\sigma}$ a locally compact group, $(\lambda, g) \rightarrow \lambda V_{g}$ is a continuous representation of this group and, moreover, the Haar measure in $G^{\sigma}$ is the product of the Haar measures of $G$ and $S_{1}$. The Haar measures of $G$ and $S_{1}$, because of their compactness, are finite and therefore the Haar measure of $G^{\sigma}$ is finite too. But a locally compact group with finite Haar measure must be compact $([5], \S 8)$ and therefore we arrived at a continuous representation $(\lambda, g) \rightarrow \lambda V_{g}$ of the compact group $G^{\sigma}$.

For such representations it is known that the Hilbert space of representation splits into a sum of finite dimensional invariant subspaces and thus the Lemma follows from proportionality of $U_{g}$ and $\lambda V_{g}$.

Remark. In fact the use of the results of Ref. [4] about connection of Borel structure and topology in separable groups is not essential. After introducing in $G^{\sigma}$ the product measure and product Borel structure we see that $G^{\sigma}$ becomes a Borel group with a left and right invariant finite measure and $(\lambda, g) \rightarrow \lambda V_{g}$ is a measurable representation of $G^{\sigma}$ in a separable Hilbert space. But for such representations the existence of a finite dimensional invariant subspace may be proved directly. I have not found the proof published but it proceeds, with minor changes, as for compact groups. As it is rather long, it is not given here.

3. Let us define $N_{k}=a_{k}^{+} a_{k} ;\left\{N_{k}\right\}$ is a commuting set of projectors Now we show that there exists in $\mathscr{H}$ a common eigenvector for all $N_{k}, k=1,2, \ldots$

It is easy to verify that for $z=e^{i t} \in S_{1}: e^{i t N_{k}} a_{k}^{+} e^{-i t N_{k}}=z a_{k}^{+}$and $e^{i t N_{k}} a_{y}^{+} e^{-i t N_{k}}=\bar{z} a_{j}^{+}$for $j \neq k$. If therefore, $p_{k}$ denote the injection $S_{1} \rightarrow G$ for which $p_{k}(z)$ has the $k-t h$ component equal to $z$ and the remaining equal to one then $e^{i t N_{h}}$ must be proportional to $U_{p k(z)}$ (they implement the same canonical transformation). 
From the proceding section we have that there exists $\mathscr{H}^{\prime}-$ a finite dimensional subspace of $\mathscr{H}$ such, that $e^{i t N_{k}} \mathscr{H}^{\prime} \subset \mathscr{H}^{\prime} .\left\{e^{i t N_{k}} \mid \mathscr{H}^{\prime}\right\}_{t, k}$ is a commuting family of unitary operators in a finite dimensional space and therefore there exists in $\mathscr{H}^{\prime}$ common eigenvector for this family. The same vector is of course a common eigenvector for $\left\{N_{k}\right\}$.

4. Let us consider in more detail such irreducible representations of canonical anticommutation relations for which there exists common eigenvector $x$ for $\left\{N_{k}\right\} . N_{k}$ are projectors and therefore $N_{k} x=0$ or $N_{k} x=x$.

Let $\mathscr{N}_{0}(x)=\left\{k: N_{k} x=0\right\}$ and $\mathscr{N}_{1}(x)=\left\{k: N_{k} x=x\right\}$. Let $a^{+}$and $a^{\prime \prime}+$ be irreducible representations in $\mathscr{H}^{\prime}$ and $\mathscr{H}^{\prime \prime}$ respectively. Suppose that $x^{\prime} \in \mathscr{H}^{\prime}$ and $x^{\prime \prime} \in \mathscr{H}^{\prime \prime}$ are common eigenvectors for $\left\{N_{k}^{\prime}\right\}$ and $\left\{N_{k}^{\prime \prime}\right\}$. Then:

The representations $a^{\prime+}$ and $a^{\prime \prime}+$ are equivalent if, and only if, the set $\mathscr{N}_{0}\left(x^{\prime}\right) \cap \mathscr{N}_{1}\left(x^{\prime \prime}\right)$ is finite.

For proof let us suppose that the set $\mathscr{N}_{0}\left(x^{\prime}\right) \cap \mathscr{N}_{1}\left(x^{\prime \prime}\right)$ is not finite and let $\left\{i_{1}, i_{2}, \ldots\right\}$ be some enumeration of its elements. Let us define:

$$
\bar{N}_{k}^{\prime}=\frac{1}{k}\left(N_{i_{1}}^{\prime}+\cdots+N_{i_{k}}^{\prime}\right) \text { and } \bar{N}_{k}^{\prime \prime}=\frac{1}{k}\left(N_{i_{1}}^{\prime \prime}+\cdots+N_{i_{k}}^{\prime \prime}\right) \text {. }
$$

Calculations give: $\left\|\bar{N}_{k}^{\prime}\right\| \leqq 1$ and $\left\|\left[a_{i}^{\prime}, \bar{N}_{k}^{\prime}\right]\right\| \leqq 1 / k$. Therefore, if for some vector $x \in \mathscr{H}^{\prime}$ there exists the strong limit $s-\lim _{k \rightarrow \infty} \bar{N}_{k}^{\prime} x$ then the sequence $\bar{N}_{k}^{\prime}$ is strongly convergent and the limit is a scalar operator. But $\bar{N}_{k}^{\prime} x^{\prime}=0$ hence $s-\lim _{k \rightarrow \infty} \bar{N}_{k}^{\prime}=0$. Applying the same to $\bar{N}_{k}^{\prime \prime}$ we see that $s-\lim _{k \rightarrow \infty} \bar{N}_{k}^{\prime \prime}=I$.

Now if there exists such unitary $U: \mathscr{H}^{\prime \prime} \rightarrow \mathscr{H}^{\prime}$ that $U a^{\prime}+(f) U^{-1}$ $=a^{\prime \prime}+(f), f \in H$, then: $U N_{k}^{\prime} U^{-1}=N_{k}^{\prime \prime}, U \bar{N}_{k}^{\prime} U^{-1}=\bar{N}_{k}^{\prime \prime}$ and therefore $U s-\lim _{k \rightarrow \infty} \bar{N}_{k}^{\prime} U^{-1}=s-\lim _{k \rightarrow \infty} \bar{N}_{k}^{\prime \prime}$, thus, we arrived to a contradiction.

The proof of the "if" part is straightforward (see also the next section).

The representations described in this section are called in [6]: the translated canonical representations.

5. In this section we prove a converse to the Friedrichs' theorem formulated in the introduction.

From Section 3, we know that there exists a common eigenvector $x$ for $\left\{N_{k}\right\}$. Let $\mathscr{N}_{0}(x)$ and $\mathscr{N}_{1}(x)$ be as in the preceding section. Let us first observe that if $i \in \mathscr{N}_{1}(x)$ then $a_{i}^{+} x=0$, because of $a_{i}^{+} N_{i}=0$, and $a_{i}^{-} x \neq 0$, because of $\left(a_{i}^{+} a_{i}^{-}+a_{i}^{-} a_{i}^{+}\right) x=x$.

Therefore if $\mathscr{N}_{1}(x)$ is finite: $\mathscr{N}_{1}(x)=\left\{i_{1}, \ldots, i_{k}\right\}$ and $x^{\prime}=a_{i_{1}}^{+} \ldots a_{i_{k}}^{+} x$ then $x^{\prime} \neq 0, \mathscr{N}_{1}\left(x^{\prime}\right)$ is an empty set and $a_{i}^{-} x=0$ for all $i$. Thus our representation is a Fock one. Similarly, if $\mathscr{N}_{0}(x)$ is finite we have an anti-Fock representation. 
But another possibility leads to a contradiction. For, let $\mathscr{N}_{0}(x)$ $=\left\{i_{1}, i_{2}, \ldots\right\}$ and $\mathscr{N}_{1}(x)=\left\{j_{1}, j_{2}, \ldots\right\}$ both be infinite. Let $A$ be such a unitary operator in $H$ that $A f_{i_{k}}=f_{j_{k}}, A f_{j_{k}}=f_{i_{k}}$ and let $B:=0$. Then the pair $A, B$ defines a canonical transformation and from assertion of the theorem to be proved there exists unitary operator $U \in \mathscr{L}(\mathscr{H})$ such, that:

$$
a(A f)=U a(f) U^{-1}, \quad f \in H .
$$

Let $\bar{N}_{k}^{\prime}=1 / k\left(N_{i_{1}}+\cdots+N_{i_{k}}\right)$ and $\bar{N}_{k}^{\prime \prime}=1 / k\left(N_{j_{1}}+\cdots+N_{j_{k}}\right)$. As in Section 4, we arrive at a contradiction by: $s-\lim _{k \rightarrow \infty} \bar{N}_{k}^{\prime}=0, s-\lim _{k \rightarrow \infty}$ $\bar{N}_{k}^{\prime \prime}=I$ and $U \bar{N}_{k}^{\prime} U^{-1}=\bar{N}_{k}^{\prime \prime}$.

Theorem is therefore proved.

The author wishes to thank Professor Amnon Katz for remarks concerning the manuscript.

\section{References}

1. Friedrichs, K. O.: Mathematical aspects of the quantum theory of fields. New York: Interscience Publishers, Inc., 1953.

2. AraKI, H., and W. Wyss: Representations of canonical anticommutation relations. Helv. Phys. Acta 37, 136 (1964).

3. Dixrmirer, J.: Les algèbres d'opérateurs dans l'espace hilbertien. Paris: GauthierVillars 1957.

4. MAckey, G. W.: Unitary representations of group extensions. Acta Math. 99, 265 (1958).

5. WeIr, A.: L'intégration dans les groupes topologiques et ses applications. $2^{e}$ ed., Acta Sci. Ind., No. 1145, Paris: Herman 1953.

6. Garding, L., and A. Wightman: Representations of the anticommutation relations. Proc. Natl. Acad. Sci. U.S. 617 (1954).

\section{J. SLAwNY}

Department of Nuclear Physics

Weizmann Institute of Science

Rehovot, Israel 